\title{
Paths to Self-Organized Criticality
}

\author{
Ronald Dickman ${ }^{1}$, Miguel A. Muñoz ${ }^{2}$, Alessandro Vespignani ${ }^{3}$, and Stefano Zapperi ${ }^{4}$ \\ ${ }^{1}$ Departamento de Física, ICEx, Universidade Federal de Minas Gerais, \\ Caixa Postal 702, \\ 30161-970 Belo Horizonte, MG, Brazil \\ ${ }^{2}$ Institute Carlos I for Theoretical and Computational Physics \\ and Departamento de Electromagnetismo y Física de la Materia \\ 18071 Granada, Spain. \\ ${ }^{3}$ The Abdus Salam International Centre for Theoretical Physics (ICTP) P.O. Box 586, 34100 Trieste, Italy \\ ${ }^{4}$ PMMH - Ecole de Physique et Chimie Industrielles, 10, rue Vauquelin, 75231 Paris CEDEX 05, France
}

Received 15 October 1999

\begin{abstract}
We present a pedagogical introduction to self-organized criticality (SOC), unraveling its connections with nonequilibrium phase transitions. There are several paths from a conventional critical point to SOC. They begin with an absorbing-state phase transition (directed percolation is a familiar example), and impose supervision or driving on the system; two commonly used methods are extremal dynamics, and driving at a rate approaching zero. We illustrate this in sandpiles, where SOC is a consequence of slow driving in a system exhibiting an absorbing-state phase transition with a conserved density. Other paths to SOC, in driven interfaces, the Bak-Sneppen model, and selforganized directed percolation, are also examined. We review the status of experimental realizations of SOC in light of these observations.
\end{abstract}

\section{Introduction}

The label "self-organized" is applied indiscriminately in the current literature to ordering or pattern formation amongst many interacting units. Implicit is the notion that the phenomenon of interest, be it scale invariance, cooperation, or supra-molecular organization (e.g., micelles), appears spontaneously. That, of course, is just how the magnetization appears in the Ising model; but we don't speak of "self-organized magnetization." After nearly a century of study, we've come to expect the spins to organize; the zero-field magnetization below $T_{c}$ is no longer a surprise. More generally, spontaneous organization of interacting units is precisely what we seek, to explain the emergence of order in nature. We can expect many more surprises in the quest to discover what kinds of order a given set of interactions lead to. All will be self-organized, there being no outside agent on hand to impose order!

"Self-organized criticality" (SOC) carries greater specificity, because criticality usually does not happen spontaneously: various parameters have to be tuned to reach the critical point. Scale-invariance in natural systems, far from equilibrium, isn't explained merely by showing that the interacting units can exhibit scale invariance at a point in parameter space; one has to show how the system is maintained (or maintains itself) at the critical point. (Alternatively one can try to show that there is generic scale invariance, that is, that criticality appears over a region of parameter space with nonzero measure $[1,2]$.) "SOC" has been used to describe spontaneous scale invariance in general; this would seem to embrace random walks, as well as fractal growth [3], diffusive annihilation ( $A+A \rightarrow 0$ and related processes), and nonequilibrium surface dynamics [4]. Here we restrict the term to systems that are attracted to a critical (scale-invariant) stationary state; the chief examples are sandpile models [5]. Another class of realizations, exemplified by the Bak-Sneppen model [6], involve extremal dynamics (the unit with the extreme value of a certain variable is the next to change). We will see that in many examples of SOC, there is a choice between global supervision (an odd state of affairs for a "self-organized" system), or a strictly local dynamics in which the rate of one or more processes must be tuned to zero.

The sandpile models introduced by Bak, Tang and Wiesenfeld (BTW) [5], Manna [7], and others have attracted great interest, as the first and clearest examples of self-organized criticality. In these models, grains of "sand" are injected into the system and are lost at the boundaries, allowing the system to reach a stationary 
state with a balance between input and output. The input and loss processes are linked in a special way to the local dynamics, which consists of activated, conservative, redistribution of sand. In the limit of infinitely slow input, the system displays a highly fluctuating, scale-invariant avalanche-like pattern of activity. One may associate rates $h$ and $\epsilon$, respectively, with the addition and removal processes. We have to adjust these parameters to realize SOC: it appears in the limit of $h$ and $\epsilon \rightarrow 0^{+}$with $h / \epsilon \rightarrow 0[1,8,9,10]$. (The addition and removal processes occur infinitely slowly compared to the local redistribution dynamics, which proceeds at a rate of unity. Loss is typically restricted to the boundaries, so that $\epsilon \rightarrow 0$ is implicit in the infinite-size limit.)

Questions about SOC fall into two categories. First, Why does self-organized criticality exist? What are the conditions for a model to have SOC? Second, the many questions about critical behavior (exponents, scaling functions, power-spectra, etc.) of specific models, and whether these can be grouped into universality classes, as for conventional phase transitions both in and out of equilibrium. Answers to the second type of question come from exact solutions [11], simulations [12], renormalization group analyses [13], and (one may hope) field theoretical analysis. Despite these insights, assertions in the literature about spontaneous or parameterfree criticality have tended to obscure the nature of the phase transition in sandpiles, fostering the impression that SOC is a phenomenon sui generis, inhabiting a different world than that of standard critical phenomena. In this paper we show that SOC is a phase transition to an absorbing state, a kind of criticality that has been well studied, principally in the guise of directed percolation [14]. Connections between SOC and an underlying conventional phase transition have also been pointed out by Narayan and Middleton [15], and by Sornette, Johansen and Dornic [16].

Starting with a simple example (Sec. II), we will see that the absorbing-state transition provides the mechanism for SOC (Sec. III). That is, we explain the existence of SOC in sandpiles on the basis of a conventional critical point. In Sec. IV we discuss the transformation of a conventional phase transition to SOC in the contexts of driven interfaces, a stochastic process that reproduces the stationary properties of directed percolation, and the Bak-Sneppen model. We find that criticality requires tuning, or equivalently, an infinite timescale separation. With this essential point in mind, we present a brief review of the relevance of SOC models to experiments in Sec. V. Sec. VI presents a summary of our ideas. We note that this paper is not intended as a complete review of SOC; many interesting aspects of the field are not discussed.

\section{A simple example}

We begin with a simple model of activated random walkers (ARW). Each site $j$ of a lattice (with periodic boundary conditions) harbors a number $z_{j}=0,1,2 \ldots$ of random walkers. (For purposes of illustration the ring $1, \ldots, L$ will do.) Initially, $N$ walkers are distributed randomly amongst the sites. Each walker moves independently, without bias, to one of the neighboring sites (i.e., from site $j$ to $j+1$ or $j-1$, with site $L+1 \equiv 1$ and $0 \equiv L$ ), the only restriction being that an isolated walker (at a site with $z_{j}=1$ ) is paralyzed until such time as another walker or walkers joins it. The active sites (with $z_{j} \geq 2$ ) follow a Markovian (sequential) dynamics: each active site loses, at a rate 1 , a pair of walkers, which jump independently to one of the neighbors of site $j$. (Thus in one dimension there is a probability of $1 / 2$ that each neighbor gains one walker, while with probability $1 / 4$ both walkers hop to the left, or to the right.)

The model we have just defined is characterized by the number of lattice sites, $L^{d}$, and the number of particles, $N$. It has two kinds of configurations: active, in which at least one site has two or more walkers, and absorbing, in which no site is multiply occupied, rendering all the walkers immobile [17]. For $N>L^{d}$ only active configurations are possible, and since $N$ is conserved, activity continues forever. For $N \leq L^{d}$ there are both active and absorbing configurations, the latter representing a shrinking fraction of configuration space as the density $\zeta \equiv N / L^{d} \rightarrow 1$. Given that we start in an active configuration (a virtual certainty for an initially random distribution with $\zeta>0$ and $L$ large), will the system remain active indefinitely, or will it fall into an absorbing configuration? For small $\zeta$ it should be easy for the latter to occur, but it seems reasonable that for sufficiently large densities $($ still $<1$ ), the likelihood of reaching an absorbing configuration becomes so small that the walkers remain active indefinitely. In other words, we expect sustained activity for densities greater than some critical value $\zeta_{c}$, with $\zeta_{c}<1$.

A simple mean-field theory provides a preliminary check of this intuition. Consider activated random walkers in one dimension. For a site to gain particles, it must have an active $(z \geq 2)$ nearest neighbor. Since active sites release a pair of walkers at a rate of unity, a given site receives a single walker from an active neighbor at rate $1 / 2$, and a pair of walkers at rate 1/4. Thus the rate of transitions that take $z_{j}$ to $z_{j}+1$ is $\left[P\left(z_{j}, z_{j+1} \geq 2\right)+P\left(z_{j}, z_{j-1} \geq 2\right)\right] / 2$; transitions from 
$z_{j}$ to $z_{j}+2$ occur at half this rate. In the mean-field approximation we ignore correlations between different sites, and factorize the joint probability into a product: $P\left(z, z^{\prime} \geq 2\right)=\rho_{z} \rho_{a}$, where $\rho_{z}$ is the fraction of sites with occupation $z$ and $\rho_{a}=\sum_{z \geq 2} \rho_{z}$ is the fraction of active sites. Using this factorization, we can write a set of equations for the site densities:

$$
\frac{d \rho_{z}}{d t}=\rho_{a}\left(\rho_{z-1}-\rho_{z}\right)+\frac{1}{2} \rho_{a}\left(\rho_{z-2}-\rho_{z}\right)+\rho_{z+2}-\theta_{z-2} \rho_{z}, \quad(z=0,1,2 \ldots),
$$

where $\theta_{n}=0$ for $n<0$ and is one otherwise. The final two terms represent active sites losing a pair of walkers. It is easy to see that the total probability, and the density $\zeta=\sum_{z} z \rho_{z}$ are conserved by the mean-field equations. This infinite set of coupled equations can be integrated numerically if we impose a cutoff at large $z$. (This is justified by the finding that $\rho_{z}$ decays exponentially for large $z$.) The mean-field theory predicts a continuous phase transition at $\zeta_{c}=1 / 2$. For $\zeta<\zeta_{c}$ the only stationary state is the absorbing one, $\rho_{a}=0$, while for $\zeta>\zeta_{c}$ the active-site density grows $\propto \zeta-\zeta_{c}$. A two-site approximation (in which we write equations for the fraction $\rho_{z, z^{\prime}}$ of nearest-neighbor pairs with given heights, but factorize joint probabilities involving three or more sites), yields $\zeta_{c}=0.75$.

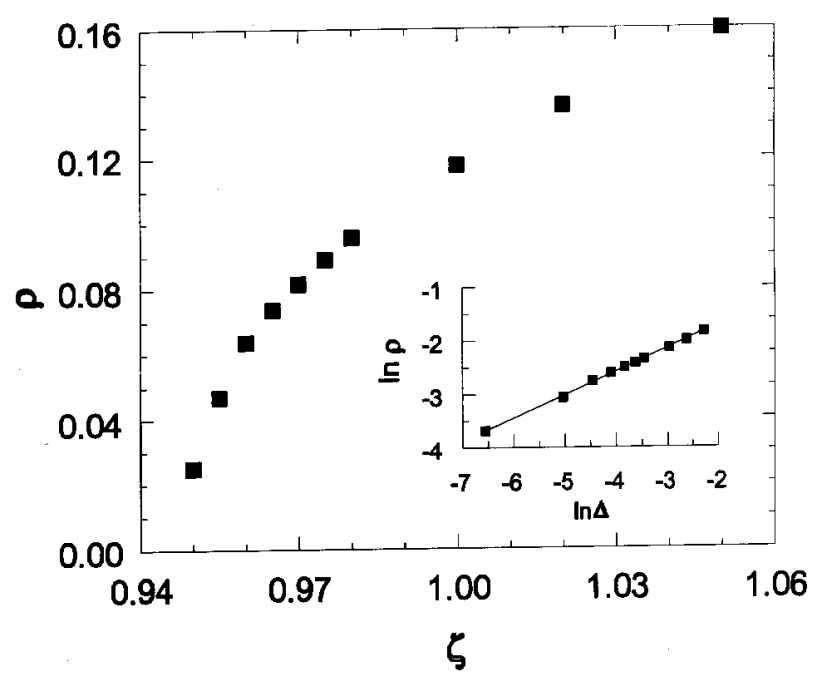

Figure 1. Stationary density $\rho$ of active sites versus density of walkers $\zeta$ in one-dimensional ARW. The inset is a logarithmic plot of the same data, where $\Delta=\zeta-\zeta_{c}$. The slope of the straight line is 0.43 .

The existence of a continuous phase transition is confirmed in Monte Carlo simulations, which yield $\zeta_{c} \simeq$ 0.9486 in one dimension, and $\zeta_{c} \simeq 0.7169$ in two dimensions. Fig. 1 shows how the stationary density of active sites $\rho_{a}$ depends on $\zeta$; we see $\rho_{a}$ growing continuously from zero at $\zeta_{c}$. (The points represent estimated densities for $L \rightarrow \infty$, based on simulation data for $L=100$
- 5000.) The inset shows that the active-site density follows a power law, $\rho_{a} \sim\left(\zeta-\zeta_{c}\right)^{\beta}$, with $\beta=0.43(1)$; a finite-size scaling analysis confirms this result [18]. In summary, activated random walkers exhibit a continuous phase transition from an absorbing to an active state as the particle density is increased above $\zeta_{c}$, with $\zeta_{c}$ strictly less than 1 . (It has yet to be shown rigorously that the active-site density in the ARW model is singular at $\zeta_{c}$, in the infinite-size limit; our numerical results are fully consistent with the existence of such a singularity.)

\section{II.1 Absorbing-State Phase Transitions}

Absorbing-state phase transitions are well known in condensed matter physics, and population and epidemic modeling [19]. The simplest example, which may be thought of as the "Ising model" of this class of systems, is the contact process [20]. Again we have a lattice of $L^{d}$ sites, each of which may be occupied (active) or vacant. Occupied sites turn vacant at a rate of unity; vacant sites become occupied at a rate of $(\lambda / 2 d) n_{0}$ where $n_{0}$ is the number of occupied nearest neighbors (the factor $2 d$ represents the number of nearest neighbors). There is a unique absorbing configuration: all sites vacant. For $\lambda$ sufficiently small, the system will eventually fall into the absorbing state, while for large $\lambda$ an active stationary state can be maintained. Letting $\rho$ represent the density of occupied sites, the mean-field theory analogous to the one formulated above for activated random walkers reads:

$$
\frac{d \rho}{d t}=(\lambda-1) \rho-\lambda \rho^{2} .
$$

This predicts a continuous phase transition (from $\rho \equiv 0$ to $\rho=1-\lambda^{-1}$ in the stationary state) at $\lambda_{c}=1$. Rigorous analyses [21, 22] confirm the existence of a continuous phase transition at a critical value $\lambda_{c}$, in any dimension $d \geq 1$. Simulations and series analyses yield $\lambda_{c}=3.29785(2)$ in one dimension. This model, and its continuous-update counterpart, directed percolation (DP; see Sec. IV), have been studied extensively. 
The critical exponents are known to good precision for $d=1,2$, and 3 ; the upper critical dimension $d_{c}=4$. There is, in addition, a well established field theory for this class of models [23, 24]:

$$
\frac{\partial \rho}{\partial t}=\nabla^{2} \rho-a \rho-b \rho^{2}+\eta(x, t) .
$$

Here $\rho(x, t)$ is a local particle density, and $\eta(x, t)$ is a Gaussian noise with autocorrelation

$$
\left\langle\eta(x, t) \eta\left(x^{\prime}, t^{\prime}\right)\right\rangle=\Gamma \rho(x, t) \delta\left(x-x^{\prime}\right) \delta\left(t-t^{\prime}\right) .
$$

That $\left\langle\eta^{2}\right\rangle$ is linear in the local density follows from the fact that the numbers of events (creation and annihilation) in a given region are Poissonian random variables, so that the variance equals the expected value. (The noise must vanish when $\rho=0$ for the latter to be an absorbing state!) This field theory serves as the basis for a strong claim of universality [23, 25]: Continuous phase transitions to an absorbing state fall generically in the universality class of directed percolation. (It is understood that the models for which we expect DP-like behavior have short-range interactions, and are not subject to special symmetries or conservation laws beyond the simple translation-invariance of the contact process. Models subject to a conservation law are known to have a different critical behavior [26].)

The activated random walkers model resembles the contact process in having an absorbing-state phase transition. We should note, however, two important differences between the models. First, ARW presents an infinite number $\left(2^{L^{d}}\right.$, to be more precise) of absorbing configurations, while the $\mathrm{CP}$ has but one. In fact, particle models in which the number of absorbing configurations grows exponentially with the system size have also been studied intensively. The simplest example is the pair contact process, in which both elementary processes (creation and annihilation) require the presence of a nearest-neighbor pair of particles [27]. In one dimension, a pair at sites $i$ and $i+1$ can either annihilate, at rate $p$, or produce a new particle at either $i-1$ or $i+2$, at rate $1-p$ (provided the selected site is vacant). This model shows a continuous phase transition from an active state for $p<p_{c}$ to an absorbing state above $p_{c}$. The static critical behavior again belongs to the DP universality class, but the critical exponents associated with spreading of activity from an initially localized region are nonuniversal, varying continuously (in one dimension) with the particle density in the surrounding region [28].

A second important difference between ARW and the CP and PCP is that the former is subject to a conservation law (the number of walkers cannot change from its initial value). In a field-theoretic description of ARW we will therefore need (at least) two fields: the local density $\rho(x, t)$ of active sites, and the local particle density $\zeta(x, t)$; the latter is frozen in regions where $\rho=0$. The evolution of $\rho$ is coupled to $\zeta$ because the particle density controls existence and level of activity in the ARW model.

Given that absorbing-state phase transitions fall generically in the universality class of directed percolation, it is natural to ask whether this is the case for activated random walkers as well. The answer, apparently, is "No." The critical exponent $\beta$ for ARW is, as we noted above, 0.43 , while for one-dimensional DP $\beta=0.2765$ [29]; the other critical exponents differ as well [18]. While the reason for this difference is not understood, it appears, at least, to be consistent with the existence of a conserved field in ARW.

To summarize, our simple model of activated random walkers has an absorbing-state phase transition, as does the contact process, directed percolation and the PCP. All possess the same basic phase diagram: active and inactive phases separated by a continuous phase transition at a critical value of a "temperature-like" parameter ( $\zeta$ in ARW, $\lambda$ in the CP). But ARW possesses an infinite number of absorbing configurations, and the evolution of its order parameter (the activesite density) is coupled to a conserved density $\zeta$. The latter presumably underlies its belonging to a different universality class than DP.

\section{Activated Random Walkers and Sandpiles}

The activated random walkers model possesses a conventional critical point: we have to tune the parameter $\zeta$ to its critical value. What has it got to do with self-organized criticality? The answer is that ARW has essentially the same local dynamics as a model known to exhibit SOC, namely, the Manna sandpile [7]. In Manna's sandpile, the redistribution dynamics runs in parallel: at each time step, all of the sites with $z \geq 2$ simultaneously liberate two walkers, which jump randomly to nearest neighbor sites. This may result in a new set of active sites, which relax at the next time step, and so on. (Time advances by one unit at each lattice update, equivalent to the unit relaxation rate of an active site in ARW.) We defined ARW with sequential dynamics as this makes it a Markov process with local transitions in configuration space, like a kinetic Ising model. There is of course nothing wrong in defining ARW with parallel dynamics; it too has an absorbing-state phase transition. 
There is a much more fundamental difference between the Manna sandpile and the ARW model: the former allows addition and loss of walkers. Recall that we defined the ARW with periodic boundary conditions; walkers can never leave the system. In the sandpile walkers may exit from one of the boundary sites. (On the square lattice, for example, a walker at an edge site has a probability of $1 / 4$ to leave the system at the next step.) If we allow walkers to leave, then eventually the system will reach an absorbing configuration. When this happens, we add a new walker at a randomly chosen site. This innocent-sounding prescription - add a walker when and only when all other activity ceases - carries the infinite time scale separation essential to the appearance of SOC in sandpiles. The sequence of active configurations between two successive additions is known as an avalanche; avalanches may involve any number of sites, from zero (no topplings) up to the entire system.

Manna showed that his model reaches a stationary state in which avalanches occur on all scales, up to the size of the system, and follow a power-law distribution, $P(s) \sim s^{-\tau}$, for $s \ll s_{c}$. (Here $s$ is the number of transfer or toppling events in a given avalanche, and $s_{c} \sim L^{D}$ is a cutoff associated with the finite system size.) In other words, the Manna sandpile, like the models devised by Bak, Tang and Wiesenfeld and others, exhibits scale invariance in the stationary state.

We know that ARW, which has the same local dynamics as the Manna sandpile, shows scale invariance when (and only when) the density $\zeta=\zeta_{c}$. So in the stationary state of the Manna model, the density is somehow attracted to its critical value. How does it happen? The mechanism of SOC depends upon a particular relation between the input and loss processes, and the conventional absorbing-state phase transition in the model with a fixed number of particles. Walkers cannot enter the system while it is active, though they may of course leave upon reaching the boundary. In the presence of activity, then, $\zeta>\zeta_{c}$ and $d \zeta / d t<0$. In the absence of activity there is addition, but no loss of walkers, so $\zeta<\zeta_{c}$ implies $d \zeta / d t>0$. Evidently, the only possible stationary value for the density in the sandpile is $\zeta_{c}$ ! Of course, it is possible to have a low level of activity locally, in a region with $\zeta<\zeta_{e}$, but under such conditions activity cannot propagate or be sustained. (One can similarly construct absorbing configurations with $\zeta>\zeta_{c}$, but these are unstable to addition of walkers, or the propagation of activity from outside.) In the infinite-size limit, the stationary activity density is zero for $\zeta<\zeta_{c}$, and positive for $\zeta>\zeta_{c}$, ensuring that $\zeta$ is pinned at $\zeta_{c}$, when loss is contingent upon activity, and addition upon its absence.
That the Manna sandpile, in two or three dimensions, with parallel dynamics, has a scale-invariant avalanche distribution is well known [7]. Here we note that the same holds for the one-dimensional version, with random sequential dynamics. Fig. 2 shows the probability distribution for the avalanche size (the total number of topplings) when we modify ARW to include loss of walkers at the boundaries, and addition at a randomly chosen site, when the system falls into an absorbing configuration. The distribution follows a power law, $P(s) \sim s^{-\tau_{s}}$, over a wide range of avalanche sizes and durations; there is, as expected, an exponential cutoff $s_{c} \sim L_{s}^{D}$ for events larger than a characteristic value associated with the finite size of the lattice. (Our best estimates are $\tau_{s}=1.10(2)$ and $D=2.21(1)$.) The upper inset of Fig. 2 shows that the stationary density approaches $\zeta_{c}$, the location of the absorbing-state phase transition, as $L \rightarrow \infty$. It is also interesting to note that, in contrast with certain deterministic one-dimensional sandpile models [30, 31], the present example appears to exhibit finite-size scaling, as shown in the lower inset of Fig. 2.

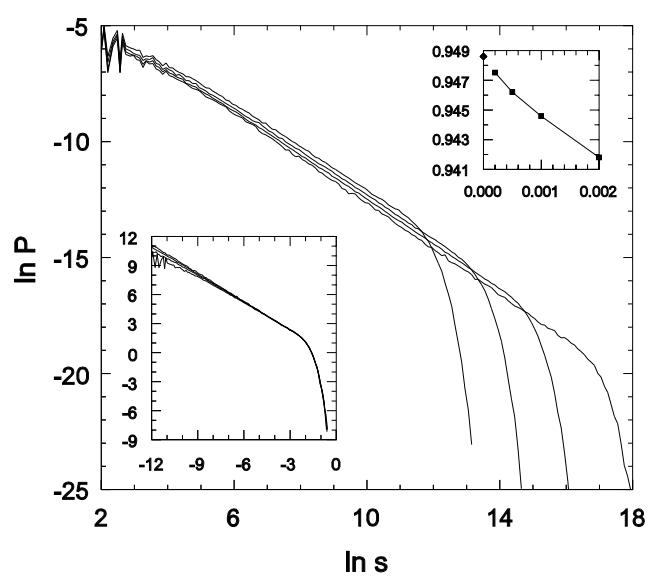

Figure 2. Stationary avalanche-size distribution in the onedimensional Manna sandpile with sequential dynamics, for $L=500,1000,2000$, and 5000 (left to right). Lower inset: finite-size scaling plot of the data in the main graph, $\ln P^{*}$ versus $\ln s^{*}$, with $s^{*} \equiv L^{-2.21} s$ and $P^{*} \equiv L^{2.43} P$. Upper inset: stationary density $\zeta$ in the inner $10 \%$ of the system, plotted versus $1 / L$. The diamond on the $\zeta$ axis is the critical density of ARW.

\section{III.1 A Recipe for SOC}

The connection between activated random walkers and the Manna sandpile suggests the following recipe for SOC. Start with a system having a continuous absorbing-state phase transition at a critical value of 
a density $\zeta$. This density should represent the global value of a local dynamical variable conserved by the $d y$ namics. Add to the conservative local dynamics (1) a process for increasing the density in infinitesimal steps $(\zeta \rightarrow \zeta+d \zeta)$ when the local dynamics reaches an absorbing configuration, and (2) a process for decreasing the density at an infinitesimal rate while the system is active. Run the system until it reaches the stationary state; it is now ready to display scale invariance.

Let's see how these elements operate in the Manna sandpile. We started with activated random walkers, which does indeed display a continuous absorbing-state transition as a function the density $\zeta$ of walkers; this density, moreover, is conserved. To this we added the input of one walker $\left(\zeta \rightarrow \zeta+1 / L^{d}\right.$ in $d$ dimensions $)$, when the system is inactive. We then broke the translational symmetry of the ARW model to define boundary sites, and allowed walkers at the boundary to leave the system. The latter implies a loss rate $d \zeta / d t \propto-L^{-1} \rho_{b}$, where $\rho_{b}$ is the activity density at the boundary sites. The conditions of our recipe are satisfied when $L \rightarrow \infty$, which we needed anyway, to have a proper phase transition in the original model.

Now we can examine the ingredients one by one. First, the phase transition in the original model should be to an absorbing state, because our input and loss steps are conditioned on the absence or presence of activity. Second, the temperature-like parameter controlling the transition should be a conserved density. So the contact process and PCP aren't suitable starting points for SOC, because the control parameter $\lambda$ isn't a dynamical variable. (To self-organize criticality in the $\mathrm{CP}$, we'd have to change $\lambda$ itself, depending on the absence of presence of activity. But this is tuning the parameter by hand!) Third, we need to change the density $\zeta$ in infinitesimal steps, else we will always be jumping between values above or below $\zeta_{e}$ without actually hitting the critical density. The same thing will happen, incidentally, if we start out with a model that has a discontinuous transition (with attendant hysteresis) between an active and an absorbing state; this yields self-organized stick-slip behavior.

The basic ingredients of our recipe are an absorbingstate phase transition, and a method for forcing the model to its critical point, by adding (removing) particles when the system is frozen (active). Following the recipe, the transformation of a conventional critical point to a self-organized one does not seem surprising [32].

\section{III.2 Firing the Baby-Sitter}

The reader may have noted a subtle inconsistency in the above discussion. We rejected the contact process as a suitable candidate for SOC because changing the parameter $\lambda$ on the basis of the current state (active or frozen) amounts to tuning. Cannot the same be said for adding walkers in the Manna sandpile? Somehow, a dynamics of walkers entering and leaving the system seems more "natural" than wholesale fiddling with a parameter. But who is going to watch for activity, to know when to add a particle? A system managed by a supervisor can hardly be called "self-organized!" If we want to avoid building a supervisor or baby-sitter into the model, we had better say that addition goes on continuously, at rate $h$, and that $S O C$ is realized in the limit $h \rightarrow 0^{+}[9,10]$. (The original sandpile definitions have a baby-sitter. Simulations, in particular, have a live-in baby-sitter to decide the next move. Addition at rate $h \rightarrow 0^{+}$is a supervisor-free interpretation of the dynamics [33].) In the recipe for SOC without baby-sitters, we replace addition (1) above with (1'): allow addition at rate $h$, independent of the state of the system, and take $h \rightarrow 0^{+}$. (There is no problem with the removal step: dissipation is associated with activity, which is local.) We pay a price when we fire the baby-sitter: there is now a parameter $h$ in the model, which has to be tuned to zero. Evidently, sandpiles don't exhibit generic scale invariance, but rather, scale invariance at a point in parameter space. This is consistent with Grinstein's definition of SOC, which requires an infinite separation of time scales from the outset [1].

\section{III.3 Variations}

In certain respects, our recipe allows greater freedom than was explored in the initial sandpile models. There is no special reason, for example, why loss of walkers has to occur at the boundaries. We simply require that activity be attended by dissipation at an infinitesimal rate. SOC has, indeed, been demonstrated in translation-invariant models with a uniform dissipation rate $\epsilon \rho$ when $\epsilon \rightarrow 0^{+}[9,34]$. In the original sandpile models, addition takes place with equal probability at any site, but restricting addition to a subset of the lattice will still yield SOC.

Our recipe allows a tremendous amount of freedom for the starting model; the only restriction is that it possess an absorbing-state critical point as a function of a conserved density. The dynamical variables can be continuous or discrete. The hopping process does not have to be symmetric, as in ARW. (In fact, directed hopping yields an exactly-soluble sandpile [35].) The model need not be defined on a regular lattice; any structure with a well defined infinite-size limit should do. The dynamics, moreover, can be deterministic. Consider 
a variant of the ARW model (on a $d$-dimensional $\mathrm{cu}^{-}$ bic lattice) in which a site is active if it has $z \geq 2 d$ walkers. At each lattice update (performed here with parallel dynamics), every active site 'topples, transferring a single walker to each of the $2 d$ nearest-neighbor sites. In this case the only randomness resides in the initial configuration. But the model again exhibits a continuous absorbing-state phase transition as we tune the number of walkers per site, $\zeta$. Starting with this deterministic model, our recipe yields the celebrated Bak-Tang-Wiesenfeld sandpile.

As a further variation, we can even relax the condition that the order parameter is coupled to a conserved field [36]. The price is the introduction of an additional driving rate. This situation is exemplified by the forestfire model $[37,38]$. The model is defined on a lattice in which each site can be in one of three states: empty, or occupied by a tree, either live or burning. Burning trees turn into empty sites, and set fire to the trees at nearest-neighbor sites, at a rate of unity. It is easy to recognize that burning trees are the active sites: any configuration without them is absorbing. In an infinite system, there will be a critical tree density that separates a phase in which fires spread indefinitely from an absorbing phase with no burning trees. In a finite system we can study this critical point by fixing the density of trees at its critical value [39].

So far we have no process for growing new trees. The forest-fire propagates like an epidemic with immunity: a site can only be active once, and there is no proper steady state [40]. As in sandpiles, to obtain a SOC state we must introduce an external driving field $f$ that introduces a small probability for each tree to catch fire spontaneously. This driving field allows the system to jump between absorbing configurations through the spreading of fires. The latter, however, are completely dissipative, i.e., the number of trees is not conserved. Thus, if we want to reach a stationary state we must introduce a second external driving field $p$ that causes new trees to appear. (Empty sites become occupied by a living tree at rate $p$.) In this case criticality is reached by the double slow driving condition $f, p \rightarrow 0$ and $f / p \rightarrow 0$. In practice, this slow driving condition is achieved by the usual supervisor, that stops fire ignition and tree growth during active intervals.

\section{III.4 Fixed-Energy Sandpiles}

If someone hands us a sandpile displaying SOC, we can identify the initial model in our recipe; it has the same local dynamics as the SOC sandpile. Thinking of the conserved $\zeta$ as an energy density, we call the starting model a fixed-energy sandpile (FES). Thus the activated random walkers model introduced in Sec. II is the fixed-energy Manna sandpile, and the variant described in the preceding subsection is the BTW FES. Now the essential feature of the fixed-energy sandpile is an absorbing-state phase transition. SOC appears when we rig up the addition and removal processes to drive the local FES dynamics to $\zeta_{c}$. To understand the details of SOC, then, we ought to try to understand the conventional phase transition in the corresponding fixed-energy sandpile. This is our program for addressing the second class of questions (about critical exponents and universality classes) mentioned in the Introduction. Since fixed-energy sandpiles have a simple dynamics (Markovian or deterministic) without loss or addition, and are translation-invariant (when defined on a regular lattice), they should be easier to study than their SOC counterparts. The relation to absorbingstate phase transitions leads to a proper identification of the order parameter [9], and suggests a strategy for constructing a field theory of sandpiles [41]. Spreading exponents, conventionally measured in absorbing-state phase transitions, are related through scaling laws to avalanche exponents, usually measured in slowly driven systems $[42,43]$.

\section{Other Paths to SOC}

\section{IV.1 Driven Interfaces}

In this section we illustrate the central idea of the preceding section - the transformation of a conventional phase transition to a self-organized one - in a different, though related, context. We begin with a single point mass undergoing driven, dissipative motion in one dimension. Its position $H(t)$ follows the equation of motion

$$
M \frac{d^{2} H}{d t^{2}}+\gamma \frac{d H}{d t}=F-F_{p}(H)
$$

where $M$ is the mass, $\gamma \dot{H}$ represents viscous dissipation, $F$ is the applied force, and $F_{p}(H)$ is a positiondependent pinning force. In many cases of interest (i.e., domain walls or flux-lines) the motion is overdamped and we may safely set $M=0$. The pinning force has mean zero $\left(\left\langle F_{p}(h)\right\rangle=0\right)$ and its autocorrelation $\left\langle F_{p}(h) F_{p}(h+y)\right\rangle \equiv \Delta(|y|)$ decays rapidly with $|y|$; the statistical properties of $F_{p}$ are independent of $H$. Assuming, as is reasonable, that $F_{p}$ is bounded $\left(F_{p} \leq F_{M}\right)$, we expect the motion to continue if the driving force $F$ exceeds $F_{M}$. Otherwise the particle gets stuck somewhere.

Now consider an elastic interface (or a flux line) subject to an external force, viscous damping, and a 
pinning force associated with irregularities in the surrounding medium. If we discretize our interface, using $H_{i}(t)$ to represent the position, along the direction of the driving force, of the $i$-th segment, the equation of motion is

$$
\gamma \frac{d H_{i}}{d t}=H_{i+1}+H_{i-1}-2 H_{i}(t)+F-F_{p, i}\left(H_{i}\right),
$$

where the $F_{p, i}\left(H_{i}\right)$ are a set of independent pinning forces with statistical properties as above. This driven interface model has a depinning transition at a critical value, $F_{e}$, of the driving force [44]. (Eq. (6) describes a linear driven interface, so-called because it lacks the nonlinear term $\propto(\nabla h)^{2}$, familiar from the KPZ equation [4, 45].) For $F<F_{c}$ the motion is eventually arrested $\left(d H_{i} / d t=0\right.$ for all $\left.i\right)$, while for $F>F_{c}$ movement continues indefinitely. Close to $F_{c}$ there are avalanche-like bursts of movement on all scales, interspersed with intervals of near-standstill. The correlation length and relaxation time diverge at $F_{c}$, as in the other examples of absorbing-state phase transitions we've discussed above. We may take the order parameter for this transition as the mean velocity, $\bar{v}=\left\langle d H_{i} / d t\right\rangle$

To reach the absorbing-state phase transition in the driven interface model we need to adjust the applied force $F$ to its critical value $F_{c}$. Can we modify this system so that it will be attracted to the critical state? Note that $F$ is not a dynamical variable, any more than is $\lambda$, in the contact process. Our sandpile recipe doesn't seem to apply here. The crucial observation is that we may change the nature of the driving, replacing the constant force $F$ with a constraint of fixed velocity, $d H_{i} / d t=v$. A finite $v$ corresponds to a state in the active phase: the mean driving force $\left.\left\langle F_{i}\right\rangle_{v}\right\rangle F_{c}$ for $v>0$. When we allow $v$ to tend to zero from above, we approach the depinning transition. This limit can be attained through an extremal dynamics in which we advance, at a given step, only the element subject to the smallest pinning force $[46,47]$. (Notice that in extremal dynamics we are directly adjusting the order parameter[16].)

To avoid the global supervision implicit in extremal dynamics we may attach each element of the interface to a spring, and move the other end of each spring at speed $V$. Now the equations of motion read

$$
\gamma \frac{d H_{i}}{d t}=H_{i+1}+H_{i-1}-2 H_{i}(t)+k\left(V t-H_{i}\right)-F_{p, i}(H)
$$

where $k$ is the spring constant. For high applied velocities, the interface will in general move smoothly, with velocity $\dot{H}=V$, while for low $V$ stick-slip motion is likely. In the overdamped regime, the amplitudes of the slips are controlled by $V$ and $k$, and the statistics of the potential. In the limit $V \rightarrow 0$, the interface motion exhibits scale invariance; $V$ plays a role analogous to $h$ in the sandpile. (The limits $V \rightarrow 0$ and $k \rightarrow 0$ have a particular significance, since the block can explore the pinning-force landscape quasistatically.) The fine tuning of $F$ to $F_{c}$ in the constant-force driving has been replaced by fine tuning $V$ to zero. This parameter tuning corresponds, once again, to an infinite timescale separation. Finally, we note that restoring inertia $(M>0)$ results in a discontinuous depinning transition with hysteresis, resulting in stick-slip motion of the sort associated with friction [48].

Once again, we have transformed an absorbing-state phase transition $\left(F=F_{c}\right.$ ) into SOC by driving the system at a rate approaching zero $(V \rightarrow 0)$. But there appear to be fundamental differences between sandpiles and driven interfaces. In the sandpile, but not in the driven interface, the order parameter is coupled to a conserved density. The sandpile, moreover, does not involve a quenched random field as does the driven interface. Despite these apparent differences, close connections have been suggested between the two kinds of model [15, 49, 50, 51]. We review this correspondence in the next subsection, following Ref. [51].

\section{IV.2 Sandpiles and Driven Interfaces}

Consider the BTW fixed-energy sandpile in two dimensions; let $H_{i}(t)$ be the number of times site $i$ has toppled since time zero. To write a dynamics for $H_{i}$, we observe that the occupation $z_{i}(t)$ of site $i$ differs from its initial value, $z_{i}(0)$, due to the inflow and the outflow of particles at this site. The outflow is given by $4 H_{i}(t)$, since each toppling expels four particles. The inflow can be expressed as $\sum_{N N} H_{j}(t)$ : site $i$ gains a particle each time one of its nearest neighbors topples. Summing the above contributions we obtain:

$$
\begin{aligned}
z_{i}(t) & =z_{i}(0)+\sum_{j N N i} H_{j}(t)-4 H_{i}(t) \\
& =z_{i}(0)+\nabla_{D}^{2} H_{i}(t)
\end{aligned}
$$


where $\nabla_{D}^{2}$ stands for the discretized Laplacian. Since sites with $z_{i}(t) \geq 4$ topple at unit rate, the dynamics of $H_{i}$ is given by

$$
\begin{aligned}
\frac{d H_{i}}{d t} & =\Theta\left[z_{i}(0)+\nabla_{D}^{2} H_{i}(t)-3\right] \\
& =\Theta\left[\nabla_{D}^{2} H_{i}(t)+F-F_{p, i}\right],
\end{aligned}
$$

where $d H_{i} / d t$ is shorthand for the rate at which the integer-valued variable $H_{i}(t)$ jumps to $H_{i}(t)+1$, and $\Theta(x)=1$ for $x>0$ and is zero otherwise. In the second line, $F \equiv \zeta-3$ and $F_{P, i} \equiv z_{i}(0)-\zeta$. (Recall that $\zeta=\left\langle z_{i}(t)\right\rangle$ for all $t$.) Thinking of $H_{i}(t)$ as a discretized interface height, Eq. (9) represents an overdamped, driven interface in the presence of columnar noise, $F_{p, i}$, which takes independent values at each site, but does not depend upon $H_{i}$, as it does in the interface model discussed in the preceding subsection. We see from this equation that tuning $\zeta$ to its critical value $\zeta_{c}$ is analogous to tuning the driving force to $F_{c}$. If we replace the discrete height $H_{i}$ in Eq. (9) with a continuous field, $H(x, t)$ (and similarly for $F_{p}$ ), and replace the $\Theta$-function by its argument, we obtain the EdwardsWilkinson surface-growth model with columnar disorder, which has been studied extensively [52]. The similarity between the present height representation and the dynamics of a driven interface suggests that the critical point of the BTW fixed-energy sandpile belongs to the universality class of linear interface depinning with columnar noise, if the rather violent nonlinearity of the $\Theta$-function is irrelevant. (The latter remains an open question. A height representation for the Manna sandpile is also possible, but is complicated by the stochastic nature of the dynamics.)

Applying the recipe of Sec. III to the driven interface, we would impose open boundaries, which drag behind the interior as they have fewer neighbors pulling on them; eventually the interface gets stuck. When this happens, we ratchet up the "force" at a randomly chosen site (in effect, $F_{p, j} \rightarrow F_{p, j}-1$ at the chosen site). The dynamics is then attracted to the critical point. Once again, we may trade supervision (checking if the interface is stuck) for a constant drive $(F \rightarrow F+h t)$ in the limit $h \rightarrow 0$.

\section{IV.3 Self-Organized Directed Percolation and the Bak-Sneppen Model}

Take the square lattice and rotate it by $45^{\circ}$, so that each site has two nearest neighbors in the row above, and two below. The sites exist in one of two states, "wet" and "dry." The states of the sites in the zeroth (top) row can be assigned at will; this defines the initial condition. A site in row $i \geq 1$ is obliged to be dry if both its neighbors in row $i-1$ are dry; otherwise, it is wet with probability $p$, and dry with probability $1-p$. This stochastic cellular automaton is called site directed percolation. Like the contact process, it possesses an absorbing state: all sites dry in row $k$ implies all dry in all subsequent rows. The dynamics of site DP can be expressed in a compact form if we define the site variable $x_{j}^{i}$ to be zero (one) if site $j$ in row $i$ is wet (dry). The variables in the next row are given by

$$
x_{j}^{i+1}=\Theta\left[\max \left\{\eta_{j}^{i}, \min \left\{x_{j-1}^{i}, x_{j+1}^{i}\right\}\right\}-p\right],
$$

where the $\eta_{j}^{i}$ are independent random variables, uniform on $[0,1]$. If both neighbors in the preceding row are in state $1, x_{j}^{i+1}$ must also equal 1 ; otherwise $x_{j}^{i+1}=0$ with probability $p$. Thinking of the rows as time slices, we see that site DP is a parallel-update version of the contact process: increasing $p$ renders the survival and propagation of the wet state more probable, and is analogous to increasing $\lambda$ in the CP. Just as the CP has a phase transition at $\lambda_{c}$, site DP has a transition from the absorbing to the active phase at $p_{c} \simeq 0.7054$.

We've already dismissed the contact process (and by extension DP) as starting models for realizing SOC via the recipe of Sec. III. Remarkably, however, it is possible to define a parameter-free stochastic process whose stationary state reproduces the properties of critical DP $[53,54,55]$. This process, self-organized directed percolation (SODP), is obtained by replacing the discrete variables in Eq. (10) by real variables which store the value of one of the previous $\eta_{j}^{i}$. In place of Eq. (10) we have simply

$$
x_{j}^{i+1}=\max \left\{\eta_{j}^{i}, \min \left\{x_{j-1}^{i}, x_{j+1}^{i}\right\}\right\},
$$

Notice that parameter $p$ has disappeared, along with the $\Theta$ function. Starting from a distribution with $x_{j}^{0}<1$ for at least one site (but otherwise arbitrary), this process eventually reaches a stationary state, characterized by the probability density $\mu(x)$. One finds that $\mu(x)$ is zero for $x<p_{c}$ (the critical value of site $\mathrm{DP}$ ), jumps to a nonzero value (infinity, in the thermodynamic limit), at $p_{\varepsilon}$, and decreases smoothly with $x$ for $x>p_{c}$. The process has discovered the critical value of site directed percolation!

Hansen and Roux explained how this works [53]: for any $p \in[0,1]$ the probability that $x_{j}^{i}<p$ is $p$ if either or both of the neighbors in the previous time slice have values less that $p$ (i.e., if the smaller of $x_{j-1}^{i-1}$ and $x_{j+1}^{i-1}$ is $<p$ ), and is zero if $x_{j-1}^{i-1}$ and $x_{j+1}^{i-1}$ both exceed $p$. This is exactly how the "wet" state propagates in site DP, with parameter $p$, if we equate the events 'site $j$ in row 
$i$ is wet' and ' $x_{j}^{i}<p$.' It follows that in the stationary state,

$$
\operatorname{Pr}\left[x_{j}^{i}<p\right]=\int_{0}^{p} \mu(x) d x,
$$

equals the probability $P(p)$ that a randomly chosen site is wet, in the stationary state of site DP with parameter $p$. This explains why $\mu(x)=0$ for $x<p_{c}$, and why $\mu\left(p_{c}\right)$ is infinite in the infinite-size limit $(d P / d p$ is infinite at $\left.p_{c}\right)$. The spatio-temporal distribution of DP is also reproduced; for example, the joint probability $\operatorname{Pr}\left[x_{j}^{i} \leq p_{c}, x_{k}^{i} \leq p_{c}\right]$ decays as a power law for large separations $|j-k|$. The process effectively studies all values of $p$ at once, greatly improving efficiency in simulations. Stochastic processes corresponding to other models (DP on other lattices, bond instead of site DP, epidemic processes) have also been devised [54, 56]. It seems unlikely, on the other hand, that such a realvalued stochastic process exists for activated random walkers or other fixed-energy sandpiles. (Of course, such a process would be of great help in studying sandpiles!)

SODP doesn't fit into the same scheme as sandpiles or driven interfaces. It is a real-valued stochastic process that generates, by construction, the probability distribution of DP for all parameter values, including $p_{c}$. The process itself does not have a phase transition; all sites are active (except those inside a sequence of 1 's - a configuration that will never arise spontaneously), since there is a finite probability for $x_{j}^{i}$ to change. SODP is self-organized in the sense that its stationary probability density has a critical singularity, without the need to adjust parameters. If we choose to regard SODP as an instance of SOC, we must recognize that the path in this case is very different from that in sandpiles or driven interfaces; the system is not being forced to its critical point by external supervision or driving. Rather, SODP is directed percolation implemented in a different (parameter-free) way. Furthermore, the dynamics embodied in Eq. (11) seems a much less realistic description of a physical system than is driven-interface motion, or even the rather artificial dynamics of a sandpile model. In the rather unlikely event that SODP were realized in a natural system, it would not immediately yield a scale-invariant "signal" such as avalanches or fractal patterns. The latter would require a second process (or an observer) capable of making fine distinctions among values of $x$ in the neighborhood of $p_{c}$. So the kind of SOC represented by SODP does not appear a likely explanation of scale invariance in nature.

A (fanciful) interpretation of Eq. (11) is that $x_{j}^{i}$ represents the "fitness" of an individual, which mates with its neighbor to produce an offspring that inherits the fitness of the less-fit parent. This offspring survives if her fitness exceeds that of an interloper, whose fitness is random. (It is, to put it crudely, as if an established population were constantly challenged by a flux of outsiders.) Seen in this light, SODP bears some resemblance to the evolutionary dynamics represented, again in very abstract form, in the Bak-Sneppen model [6]. Here, the globally minimum fitness variable, along with its nearest neighbors, is replaced by a $[0,1]$ random number at each time step. (If the $x_{j}^{i}$ are associated with different species, then the appearance of a new species at site $i$ affects the fitness of the "neighboring" species in the community in an unpredictable way.) This is a kind of extremal dynamics, a scheme we've already encountered in the driven interface model; another familiar example is invasion percolation [46]. Interestingly, the Bak-Sneppen model shows the same qualitative behavior as SODP: a singular stationary distribution of fitness values $x_{j}^{i}$. The model exhibits avalanches in which replacement of a single species provokes a large number of extinctions.

In the interface under extremal dynamics, the height $H_{i}(t)$ cannot decrease. In the Bak-Sneppen model momentary setbacks are allowed $\left(x_{j}\right.$ can decrease in a given step), but individuals of low fitness will eventually be culled. This is like an interface model with quenched noise such that, on advancing to a new position, an element may encounter a force that throws it backward, for a net negative displacement. The BakSneppen model is equivalent to a driven interface in which the least-stable site and its neighbors are updated at the same moment; we can, as before, trade extremal dynamics for a limit of infinitely slow driving.

Another way of obtaining the extremal dynamics of the Bak-Sneppen model as the limit of a stochastic process with purely local dynamics is as follows [57]. Take a one-dimensional lattice (with periodic boundaries, for definiteness), and assign random numbers $x_{j}$, independent and uniform on $[0,1]$, to each site $j=1, \ldots, L$. The configuration evolves via a series of "flips," which reset the variables at three consecutive sites. That is, when site $j$ flips, we replace $x_{j-1}, x_{j}$, and $x_{j+1}$ with three independent random numbers again drawn uniformly from $[0,1]$. Let the rate of flipping at site $j$ be $\Gamma e^{-\beta x_{j}}$, where $\Gamma^{-1}$ is a characteristic time, irrelevant to stationary properties. The Bak-Sneppen model is the $\beta \rightarrow \infty$ limit of this process.

We can get some insight into the stationary behavior via a simple analysis. Let $p(x) d x$ be the probability that $x_{j} \in[x, x+d x]$. The probability density satisfies 


$$
\frac{d p(x)}{d t}=-e^{-\beta x} p(x)-2 \int_{0}^{1} e^{-\beta y} p(x, y) d y+3 \int_{0}^{1} e^{-\beta y} p(y) d y
$$

where $p(x, y)$ is the joint density for a pair of nearestneighbor sites. If we invoke a mean-field factorization, $p(x, y)=p(x) p(y)$, then

$$
\frac{d p(x)}{d t}=-p(x)\left[e^{-\beta x}+2 I(\beta)\right]+3 I(\beta),
$$

where

$$
I(\beta) \equiv \int_{0}^{1} e^{-\beta y} p(y) d y .
$$

The stationary solution is

$$
p_{s t}(x)=\frac{3}{2} \frac{1-e^{-2 \beta / 3}}{1-e^{-2 \beta / 3}+e^{-\beta x}\left(e^{\beta / 3}-1\right)} .
$$

The solution is uniform on $[0,1]$ for $\beta=0$, as we'd expect, but in the $\beta \rightarrow \infty$ limit we have $p_{s t}=(3 / 2) \Theta(x-$ $1 / 3) \Theta(1-x)$. The probability density develops a stepfunction singularity, as in the Bak-Sneppen model. Not surprisingly, the mean-field approximation yields a rather poor prediction for the location of the singularity, which actually falls at $0.6670(1)$ [58]. (A two-site approximation places the singularity at $x=1 / 2$.) The main point is that to realize singular behavior from a local dynamics, we have to tune a parameter associated with the rates. Alternative mean-field treatments of the Bak-Sneppen model may be found in Refs. [59] and $[60]$

We can construct a model with the same local dynamics as that of Bak and Sneppen by replacing $x_{j-1}$, $x_{j}$, and $x_{j+1}$ at rate 1 , if and only if $x_{j}<r$. (Sites with $x_{j}>r$ may only change if they have a nearest neighbor below the cutoff.) In other words, only sites with $x_{j}<r$ are active; an updated site is active with probability $r$. There is an absorbing phase for small $r$, separated from an active phase by a critical point at some $r_{c}[60,61,62]$. To get the Bak-Sneppen model we forget about $r$, and declare the unique active site in the system to be the one with the smallest value of $r$. In the infinite-size limit, the probability to find a site with $r<r_{c}$ is zero, in the stationary state. We see once again that in extremal dynamics we tune the order parameter itself to zero: at each instant there is exactly one active site, so $\rho_{a}=1 / L$.
Grassberger and Zhang observed that the existence of SODP "casts doubt on the significance of selforganized as opposed to ordinary criticality." A similar doubt might be prompted by our recipe for turning a conventional critical point self-organized. Of course, even if it is possible to explain all instances of SOC in terms of an underlying conventional critical point, the details of the critical behavior remain to be understood [63]. Numerical results indicate that sandpiles, driven interfaces, and the Bak-Sneppen model define a series of new universality classes. Furthermore, no one has been able to derive the critical exponents of avalanches in SOC sandpiles, even in the abelian case, where quite a lot is known about the stationary properties [64].

\section{SOC and the Real World}

Since SOC has been claimed to be the way "nature works" [65], we would expect to find a multitude of experimental examples where this concept is useful. Originally, SOC was considered an explanation of power laws, that it provided a means whereby a system could self-tune its parameters. So once we saw a power law we could claim that it was self-generated and "explained" by SOC. The previous sections should have convinced the reader that there are no self-tuning critical points, although sometimes the fine tuning is hidden, as in sandpile models. Therefore, an "explanation" of experimentally observed power laws requires the identification of the tuning parameters controlling the scaling, as in any other ordinary critical point.

Here, we will restrict the discussion to experimental examples of avalanche behavior, leaving aside fractals and $1 / f$ noise whose connection with SOC is rather loose. (It is worth mentioning that a physical realization of self-organized criticality - without avalanches, as far as is known - has been identified in liquid ${ }^{4} \mathrm{He}$ at the $\lambda$ point [66].) Following the introduction of SOC, there were many experimental studies of avalanches, which sometimes yielded power-law distributions over a few decades, leading to endless discussions about the applicability of SOC. If we accept that self-tuned critical points don't exist, then these controversies have no basis: we have only to understand how far the system is from the critical point, and why. This task has only 
been accomplished in a few cases; several examples require further study, both experimental and theoretical.

Soon after the sandpile model was introduced, several experimental groups measured the size-distribution of avalanches in granular materials. Unfortunately, real sandpiles do not seem to be behave as the SOC sandpile model. Experiments show large periodic avalanches separated by quiescent states with only limited activity [67]. While for small piles one could try to fit the avalanche distribution with a power law over a limited range [68], the behavior would eventually cross over, on increasing the system size, to the one described above, which is not scale-invariant. The reason sand does not behave like an ideal sandpile is the inertia of the rolling grains. As grains are added, the inclination of the pile increases until it reaches the angle of maximal stability $\theta_{c}$, at which point grains start to flow. Due to inertia, the flow does not stop when the inclination falls to $\theta_{c}$, but continues until the inclination attains the angle of repose $\theta_{s}<\theta_{c}$ [69]. Since the "constant force" (i.e., with $\theta$ controlled) version of the system has a firstorder transition, it is no wonder that criticality is not observed in the slowly driven case. So if we want to see power-law avalanches we have to get rid of the inertia of the grains. Grains with small inertia exist and can be bought in any grocery store: rice! A ricepile was carefully studied in Oslo: elongated grains poured at very small rate gave rise to a convincing power-law avalanche distribution [70].

The previous discussion tells us that in order to observe a power-law avalanche distribution, inertia should be negligible. As discussed in Sec. IV, the motion of domain walls in ferromagnets and flux lines in type II superconductors is overdamped, due to eddy-current dissipation; these systems are probably the cleanest experimental examples of power-law distributed avalanches. The noise produced by domain wall motion is known as the Barkhausen effect, first detected in 1919 [71]. Since then, it has become a common non-destructive method for testing magnetic materials, and its statistical properties have been studied in detail. When the external magnetic field is increased slowly, it is possible to observe well separated avalanches, whose size distribution is a power-law over more than three decades [72-76]. Domain walls are pushed through a disordered medium by the magnetic field, so we would expect a depinning transition at some critical field $H=H_{c}$. One should note, however, that the "internal field" acting on the domains is not the external field, but is corrected by the demagnetizing field $H_{d} \simeq-N M$ where $M$ is the magnetization $[75,76]$ and $N$ the demagnetizing factor. Therefore, if we increase the external field at constant rate $c$, the internal field is given by
$H_{\text {int }}=c t-N M=c t-k y(t)$, where $y(t)$ is the average position of the domain wall and $k \propto N$. We recognize here the recipe for SOC given in section III.1: in the limit $c \rightarrow 0$ and $k \rightarrow 0$ we expect to reach the critical point. This fact was indeed verified in experiments, where $k$ can be controlled by modifying the aspect ratio of the sample [76].

In type II superconductors, when the external field is increased, flux lines are nucleated at the border of the sample and pushed inside by their mutual repulsion. The resulting flux density gradient, known as the Bean state [77], bears some analogy with sandpiles, as pointed out by De Gennes over 30 years ago [78]. Unlike sand grains, flux lines have little inertia, and exhibit power-law distributed avalanches [79]. It is still unclear whether in this system a mechanism similar to the demagnetizing field maintains a stationary avalanche state, as in ferromagnets. Simulations of flux line motion [80] have reproduced experimental results in part, but a complete quantitative explanation of the phenomenon is lacking.

Another broad class of phenomena where SOC has been invoked on several occasions is that of mechanical instabilities: fracture, plasticity and dislocation dynamics. Materials subject to an external stress release acoustic signals that are often distributed as power laws over a limited range: examples are the fracturing of wood [81], cellular glass [82] and concrete [83], in hydrogen precipitation [84], and in dislocation motion in ice crystals [85]. While it has often been claimed that these experiments provided a direct evidence of SOC, this is far from being established. In fact, fracture is an irreversible phenomenon and often the acoustic emission increases with the applied stress [81] with a sharp peak at the failure point. There is thus no stationary state in fracture, and it is debated whether the failure point can even be described as a critical point [86] or a first-order transition [87]. The situation might be different in plastic deformation, where a steady state is possible [88]; recent experimental measurements of dislocation motion appear promising [85]. We may mention some related phenomena in which avalanches have been observed, and a theoretical interpretation is still debated: martensitic transformations [89], sliding systems [90] and sheared foams [91].

Finally, it is worth mentioning that SOC has been claimed to apply to several other situations in geophysics, biology and economics. We have deliberately chosen to discuss only those examples for which experimental observations are accurate and reproducible. Even in these cases, it is often hard to distinguish between SOC-like behavior and other mechanisms for gen- 
erating power laws. This task appears almost hopeless in situations where only limited data sets are available, such as for forest fires [92], or evolution [93], and remains very complicated in other cases, such as earthquakes, as witnessed by the vast theoretical literature on the subject [94].

\section{Summary}

The genesis of self-organized criticality is a continuous absorbing-state phase transition. The dynamical system exhibiting the latter may be continuous or discrete, deterministic or stochastic, conservative or dissipative. To transform a conventional phase transition to SOC, we couple the local dynamics of the dynamical system to an external supervisor, or to a "drive" (sources and sinks with rates $\{h\}$ ). The relevant parameter(s) $\{\zeta\}$ associated with the phase transition are controlled by the supervisor or drive, in a way that does not make explicit reference to $\{\zeta\}$. One such path involves slow driving $(h \rightarrow 0)$, in which the interaction with the environment is contingent on the presence or absence of activity in the system (linked to $\{\zeta\}$ via the absorbingstate phase transition). Another, extremal dynamics, restricts activity to the least stable element in the system, thereby tuning the order parameter itself to zero. Specific realizations of this rather abstract (and general) scheme have been discussed in the preceding sections: sandpiles, forest fires, driven interfaces, and the Bak-Sneppen model.

Viewed in this light, "self-organized criticality" refers neither to spontaneous or parameter-free criticality, nor to self-tuning. It becomes, rather, a useful concept for describing systems that, in isolation, would manifest a phase transition between active and frozen regimes, and that are in fact driven slowly from outside.

\section{Acknowledgements}

We thank M. Alava, A. Barrat, A. Chessa, D.Dhar, P.L. Garrido, P. Grassberger, D. Head, K.B. Lauritsen, J. Machta, E. Marinari, R. Pastor-Satorras, L. Pietronero and A.Stella for continuous discussions and fruitful "arguments" on the significance of SOC. M.A.M., A.V., and S.Z. Acknowledge partial support from the European Network Contract No. ERBFMRXCT980183. M.A.M. also acknowledges support from the Spanish Ministerio de Educación under project DGESEIC, PB97-0842'.

\section{References}

[1] G. Grinstein, in Scale Invariance, Interfaces and Nonequilibrium Dynamics, NATO Advanced Study Institute, Series B: Physics, vol. 344, edited by A. McKane et al., (Plenum, New York, 1995).

[2] G. Grinstein, J. Appl. Phys. 69, 5441 (1991).

[3] T. Vicsek, Fractal Growth Phenomena (World Scientific, Singapore, 1992); A. Erzan, L. Pietronero and A. Vespignani, Rev. Mod. Phys. 67, 545 (1995).

[4] A. -L. Barabási and H. E. Stanley, Fractal Concepts in Surface Growth (Cambridge University Press, Cambridge, 1995).

[5] P. Bak, C. Tang and K. Wiesenfeld, Phys. Rev. Lett. 59, 381 (1987); Phys. Rev. A 38, 364 (1988).

[6] P. Bak and K. Sneppen, Phys. Rev. Lett. 71, 4083 (1993).

[7] S. S. Manna, J. Phys. A 24, L363 (1991).

[8] T. Hwa and M. Kardar, Phys. Rev. A 45, 7002 (1992).

[9] A. Vespignani and S. Zapperi, Phys. Rev. Lett. 78, 4793 (1997); Phys. Rev. E 57, 6345 (1998).

[10] R. Dickman, A. Vespignani and S. Zapperi, Phys. Rev. E 57, 5095 (1998).

[11] See the excellent review by D. Dhar, e-print condmat/9909009.

[12] Avalanche exponents: P. Grassberger and S. S. Manna, J. Phys. (France) 51, 1077 (1990); S. S. Manna, J. Stat. Phys. 59, 509 (1990); S. Lübeck and K.D. Usadel, Phys. Rev. E 55, 4095 (1997); ibid. 56, 5138 (1997). Upper critical dimension: A. Chessa, E. Marinari, A. Vespignani and S. Zapperi, Phys. Rev. E 57, R6241 (1998); S. Lübeck, ibid 56, 1590 (1997). Universality classes: A. Ben-Hur and O. Biham, Phys. Rev. E 53, R1317 (1996); A. Chessa, H. E. Stanley, A. Vespignani and S. Zapperi, Phys. Rev. E 59, R12 (1999). Multifractal properties: L. P. Kadanoff, S. R. Nagel, L. Wu and S. Zhou, Phys. Rev. A 39, 6524 (1989); M. De Menech, A. L. Stella and C. Tebaldi, Phys. Rev. E 58, R2677 (1998).

[13] A. Díaz-Guilera, Europhys. Lett. 26, 177 (1994); L. Pietronero, A. Vespignani and S. Zapperi, Phys. Rev. Lett. 72, 1690 (1994); J. Hasty and K. Wiesenfeld, J. Stat. Phys. 86, 1179 (1997).

[14] W. Kinzel, Z. Phys. B 58, 229 (1985).

[15] O. Narayan and A. A. Middleton, Phys. Rev. B 49244 (1994).

[16] D. Sornette, A. Johansen, and I. Dornic, J. Phys. I (France) 5, 325 (1995).

[17] The expert reader will have noticed that activated random walks follow the same local dynamics as the Manna sandpile [7].

[18] Details of simulations of activated random walkers are given in R. Dickman, A. Vespignani, S. Zapperi, M. A. Muñoz, and M. Alava, in preparation.

[19] R. Dickman, in Nonequilibrium Statistical Mechanics in One Dimension, edited by V. Privman, (Cambridge University Press, Cambridge 1996); G. Grinstein and M. A. Muñoz, in Fourth Granada Lectures in Computational Physics, edited by P. Garrido and J. Marro, 
Lecture Notes in Physics, 493, 223 (Springer-Verlag, Berlin, 1997). J. Marro and R. Dickman, Nonequilibrium Phase Transitions in Lattice Models (Cambridge University Press, Cambridge, 1999).

[20] T. E. Harris, Ann. Probab. 2, 969 (1974).

[21] T. M. Liggett, Interacting Particle Systems, (SpringerVerlag, New York, 1985).

[22] C. Bezuidenhout and G. Grimmett, Ann. Probab. 18, $1462(1990)$.

[23] H. K. Janssen, Z. Phys. 42, 141 (1981); ibid. 58, 311 (1985).

[24] J. Cardy, Scaling and Renormalization in Statistical Physics (Cambridge University Press, Cambridge, 1996), Ch. 10.

[25] P. Grassberger, Z. Physik. B 47, 465 (1982).

[26] This is the case of branching, annihilating random walks with even numbers of offspring, also known as the "directed Ising" universality class. See: P. Grassberger, F. Krause, and T. von der Twer, J. Phys. A 17, L105 (1984); P. Grassberger, ibid. 22, L1103 (1989); H. Takayasu and A. Yu. Tretyakov, Phys. Rev. Lett. 68, 3060 (1992); I. Jensen, Phys. Rev. E 50, 3623 (1994); N. Menyhard and G. Ódor, J. Phys. A 29, 7739 (1996); J. Cardy and U. C. Täuber, Phys. Rev. Lett. 77, 4780 (1996). H. Hinrichsen, Phys. Rev. E 55, 219 (1997); W. Hwang, S. Kwon, H. Park, and H. Park, Phys. Rev. E $\mathbf{5 7 ,} 6438$ (1998).

[27] I. Jensen, Phys. Rev. Lett. 70, 1465 (1993).

[28] I. Jensen and R. Dickman, Phys. Rev. E 48, 1710 (1993).

[29] I. Jensen, J. Phys. A 29, 7013 (1996).

[30] A. A. Ali and D. Dhar, Phys. Rev. E 51, R2705 (1995); Phys. Rev. E 52, 4804 (1995).

[31] See L. P. Kadanoff et al in Ref [12]

[32] The question of open versus closed models for SOC is also discussed in A. Montakhab and J. M. Carlson, Phys. Rev. E 58, 5608 (1998).

[33] Sometimes the supervisor holds the job description "gardener"; see H. M. Broeker and P. Grassberger, eprint: cond-mat/9902195.

[34] S. S. Manna, L. B. Kiss and J. Kertész, J. Stat. Phys. 61, $923(1990)$.

[35] D. Dhar and R. Ramaswamy, Phys. Rev. Lett. 63, 1659 (1989).

[36] J. E. S. Socolar, G. Grinstein, and C. Jayaprakash, Phys. Rev. E 47, 2366 (1993).

[37] B. Drossel and F. Schwabl, Phys. Rev. Lett. 69, 1629 (1992).

[38] S. Clar, B. Drossel and F. Schwabl, Phys. Rev. E 50, 1009 (1994).

[39] S. Clar, B. Drossel and F. Schwabl, J. Phys. C 8, 6803 (1996).

[40] Epidemics with immunity fall in the universality class of dynamic percolation. See J. L. Cardy and P. Grassberger, J. Phys. A 18, L267 (1985); H. K. Janssen, Z. Phys. B 58, 311 (1985).
[41] A. Vespignani, R. Dickman, M. A. Muñoz, and Stefano Zapperi, Phys. Rev. Lett. 81, 5676 (1998).

[42] M. A. Muñoz, R. Dickman, A. Vespignani, and Stefano Zapperi, Phys. Rev. E 59, 6175 (1999).

[43] A. Chessa, E. Marinari and A. Vespignani, Phys. Rev. Lett. 80, 4217 (1998).

[44] See H. Leschhorn, T. Nattermann, S. Stepanow and LH. Tang, Ann. Phys. 6, 1 (1997), and references therein.

[45] M. Kardar, G. Parisi, and Y.-C. Zhang, Phys. Rev. Lett 56, 889 (1986).

[46] D. Wilkinson and J. F. Willemsen, J. Phys. A 16, 3365 (1983).

[47] Extremal dynamics has been introduced in invasion percolation [46], applied to linear interface models in S. I. Zaitsev, Physica A 189, 411, (1992), and S. Roux and A. Hansen, J. Phys. (France) I, 4, 515 (1994); and to quenched KPZ in : K. Sneppen, Phys. Rev. Lett. 69, 3539 (1992); S. Havlin, A.-L. Barabási, S. V. Buldyrev, C. K. Peng, M. Schwartz, H. E. Stanley, and T. Vicsek, in Growth Patterns in Physical Sciences and Biology [Proc. 1991 NATO Advanced Research Workshop, Granada], edited by J. M. Garcia-Ruiz, E. Louis, P. Meakin, and L. M. Sander (Plenum Press, New York, 1993).

[48] B. N. J. Persson, Sliding friction, (Springer-Verlag, Berlin, 1998).

[49] O. Narayan and D. S. Fisher, Phys. Rev. B 487030 (1993).

[50] M. Paczuski and S. Boettcher, Phys. Rev. Lett. 77, 111 (1996)

[51] K. B. Lauritsen and M. Alava, e-print: condmat/9903346.

[52] G. Parisi and L. Pietronero, Europhys. Lett. 16, 321 (1991); Physica A 179, 16 (1991).

[53] A. Hansen and S. Roux, J. Phys A 20, L873 (1987).

[54] P. Grassberger and Y.-C. Zhang, Physica A 224, 169 (1996).

[55] S. Maslov and Y-C. Zhang, Physica A 223, 1 (1996).

[56] F. Bagnoli, P. Palmerini, and R. Rechtman, Phys. Rev. E 55, 3970 (1997).

[57] Similar paths have been followed by K. Sneppen, Physica A 221, 168 (1995); M. Vergeles, Phys. Rev. Lett. 75, 1969 (1995); A. Gabrielle, G Calderelli, and L. Pietronero, e-print: cond-mat/9910425.

[58] P. Grassberger, Phys. Lett. A 200, 277 (1995).

[59] H. Flyvbjerg, K. Sneppen, and P. Bak, Phys. Rev. Lett. 71,4087 (1993)

[60] M. Paczuski, S. Maslov and P. Bak, Phys. Rev. E 53 414 (1996).

[61] B. Jovanović, S. V. Buldyrev, S. Havlin, and H. E. Stanley, Phys. Rev. E 50, R2403 (1994).

[62] A. Lipowski and M. Lopata, Phys. Rev. E 60, 1516 (1999); A. Lipowski, e-print: cond-mat/9910029. 
[63] It is sometimes argued that Takayasu's aggregation model (H. Takayasu, Phys. Rev. Lett. 63, 2563 (1989)) is an example of SOC without slow driving. The model has a scale-invariant scaling limit, rather than a stationary state as such. More significantly, Dhar has shown that Takayasu's model is equivalent to a sandpile model, which does exhibit scale invariance under slow driving.

[64] See Ref. [11], which also includes a discussion of directed sandpiles [35], for which the avalanche exponents have been determined.

[65] P. Bak, How Nature works (Copernicus, New York, 1996).

[66] J. Machta, D. Candela, and R. B. Hallock, Phys. Rev. E 47, 4581 (1993).

[67] H. M. Jaeger, C. H.- Liu and S. R. Nagel, Phys. Rev. Lett. 62, 40 (1989).

[68] M. Bretz et al. Phys. Rev. Lett. 69, 2431 (1992)

[69] H. M. Jaeger, S. R. Nagel and R. P. Behringer, Rev. Mod. Phys. 68, 1259 (1996).

[70] V. Frette, K. Christensen, A.M. Malthe-Sørenssen, J. Feder, T. Jøssang and P.Meakin, Nature 397, 49 (1996).

[71] H. Barkhausen, Z. Phys. 20, 401 (1919).

[72] G. Bertotti, G. Durin, and A. Magni, J. Appl. Phys. 75, 5490 (1994).

[73] G. Durin, G. Bertotti, and A. Magni, Fractals 3, 351 (1995).

[74] D. Spasojević, S. Bukvić, S. Milosević, and H. E. Stanley, Phys. Rev. E 54, 2531 (1996).

[75] J. S. Urbach, R. C. Madison, and J. T. Markert, Phys. Rev. Lett. 75, 276 (1995).

[76] S. Zapperi, P. Cizeau, G. Durin, and H. E. Stanley, Phys. Rev. B 58, 6353 (1998).

[77] C. P. Bean, Rev. Mod. Phys. 36, 31 (1964).

[78] P.-G. de Gennes, Superconductivity of metals and alloys (Benjamin, New York, 1966).

[79] S. Field, J. Witt, F. Nori, and X. Ling, Phys. Rev. Lett. 74, 1206 (1995).

[80] C. J. Olson, C. Reichhard and F. Nori, Phys. Rev. B 56, 6175 (1997); K. E. Bassler and M. Paczuski, Phys. Rev. Lett. 81, 3761 (1998).
[81] A. Garcimartín, A. Guarino, L. Bellon and S. Ciliberto, Phys. Rev. Lett. 79, 3202 (1997); A. Guarino, A. Garcimartín and S. Ciliberto, Eur. Phys. J. B 6, 13 (1998).

[82] C. Maes, A. Van Moffaert, H. Frederix and H. Strauven, Phys. Rev. B 57, 4987 (1998).

[83] A. Petri, G. Paparo, A. Vespignani, A. Alippi and M. Costantini, Phys. Rev. Lett. 73, 3423 (1994).

[84] G. Cannelli, R. Cantelli and F. Cordero, Phys. Rev. Lett. 70, 3923 (1993).

[85] J. Weiss and. J.-R. Grasso, J. Phys. Chem. B 101, 6113 (1997).

[86] D. Sornette and J. V. Andersen, Eur. Phys. J. B 1, 353 (1998).

[87] S. Zapperi, P. Ray, H. E. Stanley and A. Vespignani, Phys. Rev. E 59, 5049 (1999).

[88] S. Zapperi, A. Vespignani and H. E. Stanley, Nature 388, 658 (1997).

[89] L. Carrillo, L. Mañosa, J. Ortín, A. Planes and E. Vives, Phys. Rev. Lett. 81, 1889 (1998).

[90] S. Ciliberto and C. Laroche, J. Phys. I (France) 4, 223 (1994).

[91] A. D. Gopal and D. J. Durian, Phys. Rev. Lett. 75, 2610 (1995)

[92] B. D. Malamud, G. Morein, and D. L. Turcotte, Science 281, 1840 (1998).

[93] Conflicting conclusions have been drawn from the same paleontologic data: R. V. Solé, S. C. Manrubia, M. Benton and P. Bak, Nature 388, 764 (1997); J. W. Kirchner and A. Weil, Nature 395, 337 (1998).

[94] See for example: K. Chen, P. Bak and S. P. Obukhov, Phys. Rev. A 43, 625 (1991); J. M. Carlson, J. S. Langer and B. E. Shaw, Rev. Mod. Phys. 66, 657 (1994); J. B. Rundle, W. Klein and S. Gross, Phys. Rev. Lett. 76, 4285 (1996); J. B. Rundle, D. L. Turcotte and W. Klein (eds.) Proceedings of the Santa Fe Institute Workshop on Reduction and Predictability of Natural Disasters, Santa Fe, NM, 1994 (Addison-Welsley, Reading, MA, 1995), and references therein. 